\title{
LFQProfiler and RNPxl - Open-source tools for label-free quantification and protein-RNA cross-linking integrated into Proteome Discoverer
}

\author{
Supporting Information
}

Johannes Veit
Timo Sachsenberg
Aleksandar Chernev
Fabian Aicheler
Henning Urlaub
Oliver Kohlbacher

\begin{abstract}
Center for Bioinformatics, University of Tübingen, Tübingen, Germany Center for Bioinformatics, University of Tübingen, Tübingen, Germany Max Planck Institute for Biophysical Chemistry, Göttingen, Germany Center for Bioinformatics, University of Tübingen, Tübingen, Germany Max Planck Institute for Biophysical Chemistry, Göttingen, Germany Center for Bioinformatics, University of Tübingen, Tübingen, Germany; Quantitative Biology Center, University of Tübingen, Tübingen, Germany; Biomolecular Interactions, Max Planck Institute for Developmental Biology, Tübingen, Germany
\end{abstract}

Table of contents:

- Supplementary Table S1: Parameter settings of PD nodes in the LFQProfiler benchmark (page S-2)

- Supplementary Figure S1: Volcano plot for LFQProfiler benchmarking results. (page S-3) 


\begin{tabular}{|c|c|c|c|}
\hline PD Node & Category & Parameter & Value \\
\hline Spectrum Selector & Scan Event Filters & MS Order & Any \\
\hline LFQProfiler FF & Feature Finding & Mass tolerance & $6 \mathrm{ppm}$ \\
\hline LFQProfiler FF & Feature Finding & Charge Low & 2 \\
\hline LFQProfiler FF & Feature Finding & Charge High & 4 \\
\hline LFQProfiler FF & Feature Finding & Typical RT & 40 \\
\hline LFQProfiler FF & Feature Finding & Minimum RT & 5 \\
\hline LFQProfiler FF & Feature Finding & Averagine similarity & 0.8 \\
\hline Sequest HT & Input Data & Protein Database & yeast_plus_ups.fasta \\
\hline Sequest HT & Input Data & Enzyme Name & Trypsin/P (full) \\
\hline Sequest HT & Tolerances & $\begin{array}{l}\text { Precursor Mass } \\
\text { Tolerance }\end{array}$ & $10 \mathrm{ppm}$ \\
\hline Sequest HT & Tolerances & $\begin{array}{l}\text { Fragment Mass } \\
\text { Tolerance }\end{array}$ & $0.6 \mathrm{Da}$ \\
\hline Sequest HT & $\begin{array}{l}\text { Dynamic } \\
\text { Modifications }\end{array}$ & $\begin{array}{l}\text { 1. Dynamic } \\
\text { Modification }\end{array}$ & $\begin{array}{l}\text { Oxidation / +15.995 } \\
\text { Da (M) }\end{array}$ \\
\hline Sequest HT & $\begin{array}{l}\text { Dynamic } \\
\text { Modifications } \\
\text { (peptide terminus) }\end{array}$ & $\begin{array}{l}\text { 1. N-terminal } \\
\text { Modification }\end{array}$ & $\begin{array}{l}\text { Acetyl / +42.011 (N- } \\
\text { Terminus) }\end{array}$ \\
\hline Sequest HT & Static Modifications & 1. Static Modification & $\begin{array}{l}\text { Carbamidomethyl / } \\
+57.021(\mathrm{C})\end{array}$ \\
\hline LFQProfiler & Feature Linking & $\begin{array}{l}\text { Perform RT } \\
\text { alignment }\end{array}$ & True \\
\hline LFQProfiler & Feature Linking & Peptide min \#runs & 5 \\
\hline LFQProfiler & Feature Linking & $\begin{array}{l}\text { Max RT difference } \\
\text { [min] }\end{array}$ & 1 \\
\hline LFQProfiler & Feature Linking & Max m/z difference & $10 \mathrm{ppm}$ \\
\hline LFQProfiler & ID mapping & $\begin{array}{l}\text { Max RT difference } \\
{[\mathrm{min}]}\end{array}$ & 0.2 \\
\hline LFQProfiler & ID mapping & Max m/z difference & $10 \mathrm{ppm}$ \\
\hline LFQProfiler & ID mapping & q-Value threshold & 0.01 \\
\hline LFQProfiler & ID mapping & Protein database & Yeast_plus_ups.fasta \\
\hline LFQProfiler & ID mapping & $\mathrm{m} / \mathrm{z}$ reference & peptide \\
\hline LFQProfiler & $\begin{array}{l}\text { Intensity } \\
\text { normalization }\end{array}$ & Method & median \\
\hline LFQProfiler & Protein quantification & Protein-level FDR & 0.01 \\
\hline LFQProfiler & Protein quantification & Use peptides & greedy \\
\hline LFQProfiler & Protein quantification & $\begin{array}{l}\text { Fido pre-filtering PEP } \\
\text { threshold }\end{array}$ & 0.2 \\
\hline LFQProfiler & Protein quantification & Top & 5 \\
\hline LFQProfiler & Protein quantification & Averaging & sum \\
\hline LFQProfiler & Protein quantification & Include all & True \\
\hline LFQProfiler & Protein quantification & Filter charge & False \\
\hline LFQProfiler & Protein quantification & Fix peptides & False \\
\hline
\end{tabular}

Supplementary Table S1: Parameter settings of PD nodes in the LFQProfiler benchmark. Parameters not mentioned here were set to default settings. 


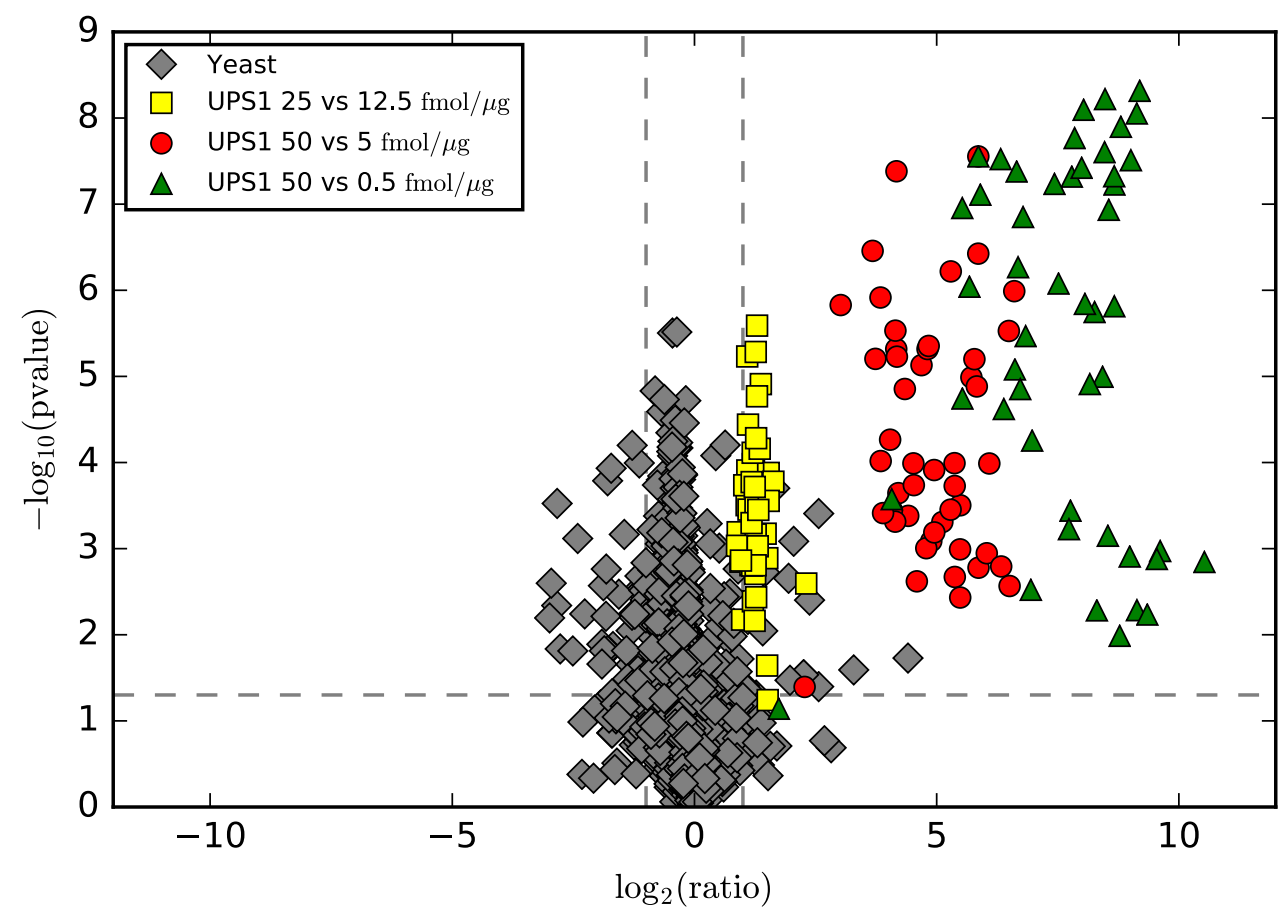

Supplementary Figure S1: Combined volcano plot for all three comparisons 50 vs 0.5 fmol / $\mu$ g (green), 50 vs $5 \mathrm{fmol} / \mu \mathrm{g}$ (red), and $25 \mathrm{vs} 12.5 \mathrm{fmol} / \mu \mathrm{g}$ (yellow) of spike-in protein standard. Yeast proteins are shown in gray. For each protein, the negative $\log _{10}$ of the p-value from Welsh's t-test for differential abundance between the two conditions is plotted against the corresponding $\log _{2}$ fold change. 